\title{
NUEVOS REGISTROS DE DASYPODIDAE (XENARTHRA) DEL MIOCENO TARDÍO DE LA PROVINCIA DE LA PAMPA, ARGENTINA
}

\author{
Graciela I. ESTEBAN', Norma L. NASIF'y \\ Claudia I. MONTALVO'
INSUGEO, Facultad de Ciencias Naturales e Instituto Miguel Lillo. Universidad Nacional de Tucumán. Miguel Lillo 205, (4000) San Miguel de Tucumán. Argentina. Pampa, Uruguay 151, (6300) Santa Rosa, La Pampa, Argentina.
e-mail:'instlillo@inforia.com.ar.2overlord@cpsarg.com \\ ? Facultad de Ciencias Exactas y Naturales, Universidad Nacional de La
}

Esteban, G.I., Nasif, N.L. y Montalvo, C.I. 2001. Nuevos registros de Dasypodidae (Xenarthra) del Mioceno tardío de la provincia de La Pampa, Argentina. [New records of Dasypodidae (Xenarthra) from the Late Miocene of the La Pampa province, Argentina.] Revista Española de Paleontología, 16(1), 77-87. ISSN 0213-6937.

\begin{abstract}
New Dasypodidae (Xenarthra, Cingulata) records from the Cerro Azul Formation in the province of La Pampa, Argentina are reported here. The material consists of isolated scutes of the dorsal carapace from Laguna Chillhué, Cerro de los Guanacos, Bajo Giuliani, Quehué, Naicó, Laguna Guatraché, Telén, Loventué, Cerro de la Bota, and Salinas Grandes de Hidalgo, between $36^{\circ}-38^{\circ} \mathrm{S}$ and $63^{\circ}-67^{\circ} \mathrm{W}$. Chasicotatus ameghinoi, Macrochorobates scalabrinii, Zaedyus pichiy, Vetelia perforata, Doellotatus inornatus, Doellotatus chapadmalensis, Proeuphractus, Macroeuphractus morenoi, Chorobates villosissimus, and Gen. nov. "A" Scillato Yané are recogniced. The first seven taxa and Gen. nov. "A" Scillato Yané are reported here for the first time from this formation as well as a possible new species of Proeuphractus. The Cerro Azul Formation is referred to the Huayquerian (Late Miocene) based on the presence of several groups of fossil mammals. Chasicotatus ameghinoi is the most modern record for this taxon, whereas the age of Zaedyus pichiy, Doellotatus inornatus, Doellotatus chapadmalensis, and Gen. nov. "A" Scillato Yané is traced back to the Huayquerian.
\end{abstract}

Keywords: Dasypodidae, Xenarthra, systematic, Late Miocene, Huayquerian, Argentina.

\section{RESUMEN}

Se dan a conocer nuevos registros de Dasypodidae (Xenarthra, Cingulata) para la Formación Cerro Azul en la provincia de La Pampa, Argentina. El material estudiado consiste en placas aisladas de la coraza dorsal y procede de las localidades: Laguna Chillhué, Cerro de los Guanacos, Bajo Giuliani, Quehué, Naicó, Laguna Guatraché, Telén, Loventué, Cerro de la Bota y Salinas Grandes de Hidalgo, comprendidas entre los $36^{\circ}-38^{\circ} \mathrm{S}$ and $63^{\circ}-67^{\circ}$ O. Se reconocen Chasicotatus ameghinoi, Macrochorobates scalabrinii, Zaedyus pichiv, Vetelia perforata. Doellotatus inornatus, Doellotatus chapadmalensis, Proeuphractus, Macroeuphractus morenoi, Chorobates villosissimus, y Gen. nov. "A" Scillato Yané. Los siete primeros taxones y Gen. nov. "A" Scillato Yané son citados por vez primera para esta formación. Se constata la presencia de una probable nueva especie de Proeuphractus. La Formación Cerro Azul es referida al Huayqueriense (Mioceno tardío) sobre la base de diferentes grupos de mamíferos fósiles. En este marco, el registro de Chasicotatus ameghinoi es el más moderno para el taxón, mientras que los de Zaedyus pichiy, Doellotatus inornatus, Doellotatus chapadmalensis y Gen. nov. "A" Scillato Yané extienden la antigüiedad de estos dasipódidos hasta el Huaycueriense.

Palabras clave: Dasypodidae, Xenarthra, sistemática, Mioceno tardío, Huayqueriense, Argentina.

\section{INTRODUCCIÓN}

Son numerosos los restos de mamíferos fósiles que se conocen del Mioceno tardío de la provincia de La Pampa
(Pascual y Bocchino, 1963; Pascual et al., 1965; Ortega Hinojosa, 1967; Pascual y Bondesio, 1982; Montalvo y Casadío, 1988; Goin y Montalvo, 1988; Visconti y Montalvo, 1990; Verzi et al., 1990; 1991; 1993; 1994; 


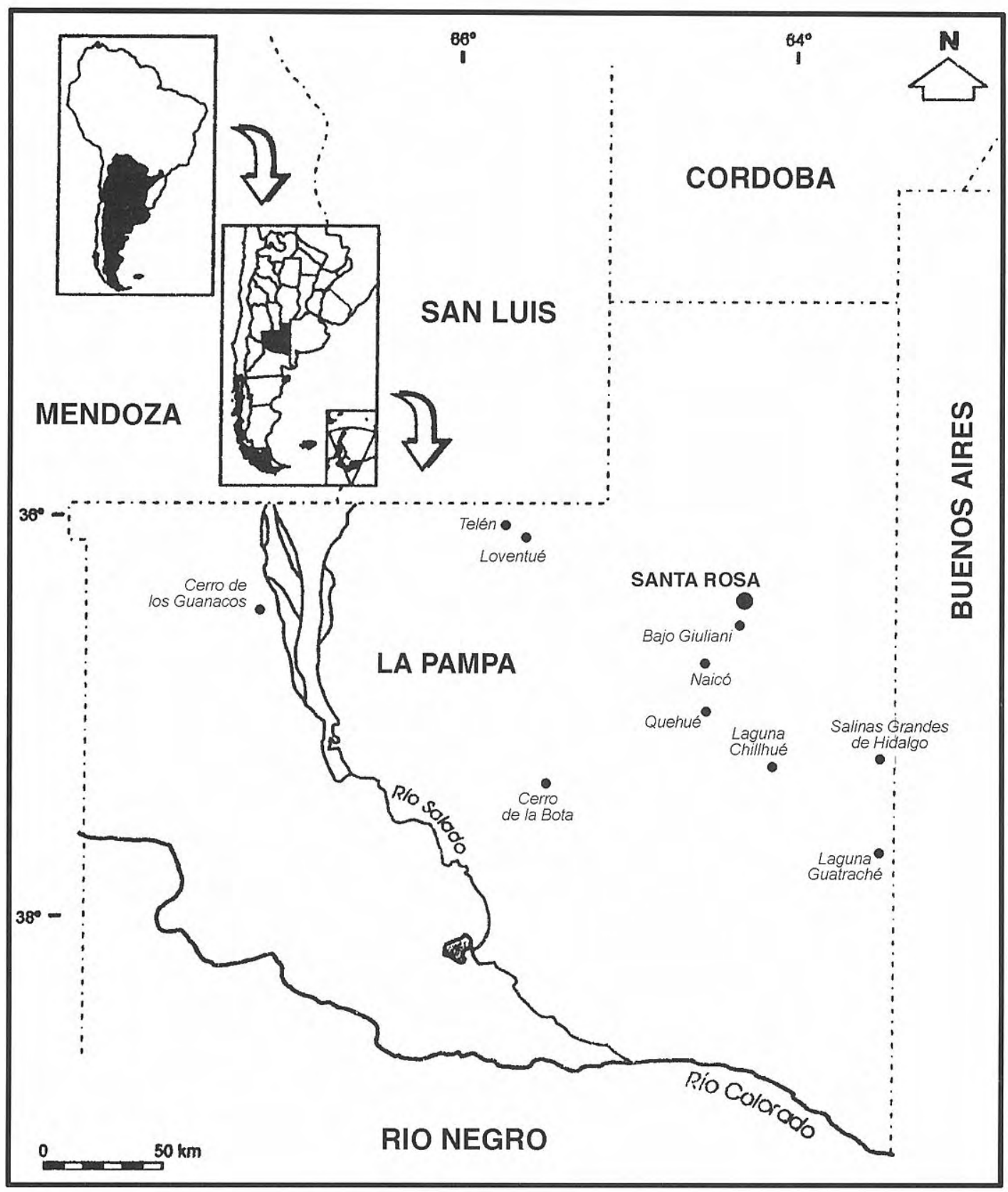

Figura 1. Mapa de ubicación de las localidades fosilíferas de las que procede el material estudiado, provincia de La Pampa, Argentina.

Map showing the fossiliferous localities where the material was recovered, La Pampa province, Argentina.

1995; 1996; 1999; Montalvo et al., 1995; 1996; 1998; Esteban et al., 1996; Goin et al., 1997 y en prensa); sin embargo, el registro de Dasypodidae es escaso. ScillatoYané (1980) sólo menciona un Eutatini -Doellotatus chapadmalensis Bordas- y tres Euphractini -Vetelia perforata Scillato-Yané, Chorobates villosissimus (Rovereto) y Macroeuphractus morenoi (Lydekker). Otras referencias a dasipódidos se citan en los trabajos inéditos de Zetti (1972) y Scillato-Yané (1982).

En el presente trabajo, se dan a conocer nuevos registros de dasipódidos de la provincia de La Pampa. Los fósiles proceden de depósitos asignados a la Formación Cerro Azul (Linares et al., 1980), que afloran en las siguientes localidades: Bajo Giuliani (Dpto. Capital), Telén y Loventué (Dpto. Loventué), Laguna Guatraché (Dpto. Hucal), Quehué y Cerro de la Bota (Dpto. Utracán), Laguna Chillhué (Dpto. Guatraché), Salinas Grandes de Hidalgo (Dpto. Atreucó), Naicó (Dpto. Toay) y Cerro de los Guanacos (Dpto. Chical Có); todas ellas comprendidas entre los $36^{\circ}-38^{\circ}$ de latitud sur y $\operatorname{los} 63^{\circ}-67^{\circ}$ de longitud oeste (Fig. 1).

El material estudiado consiste en placas aisladas de la coraza dorsal. Si bien los restos fueron hallados en niveles de la Formación Cerro Azul, en muchos casos no fue posible definir la procedencia estratigráfica precisa (material numerado con barra), ya que dada la morfología 


\begin{tabular}{|c|c|c|c|c|c|c|c|c|c|c|}
\hline TAXONES & $\mathrm{SC}$ & $\mathbf{F R}$ & V & $\mathbf{B}$ & HU & MO & CHA & MA & $\mathbf{E N}$ & $\mathbf{L U}$ \\
\hline Vetelia perforata & & & & & $\sigma 0$ & & & & & \\
\hline Macroeuphractus morenoi & & & & & & & & & & \\
\hline Macrochorobates scalabrinii & & & & & & & & & & \\
\hline Proeuphractus & & & & & & & & & & \\
\hline Zaedyus pichiy & & & & & & & & $?$ & & \\
\hline Género nov."A” Scillato Yané & & & & & & & & & & \\
\hline Chorobates villosissimus & & & & & & & & & & \\
\hline Chasicotatus ameghinoi & & & & & & & & & & \\
\hline Doellotatus inornatus & & & & & & & & & & \\
\hline Doellotatus chapadmalensis & & & & & & & & & & \\
\hline
\end{tabular}

Tabla 1. Distribución temporal de los taxones de dasipódidos estudiados. La barra continua indica los registros tomados de Scillato Yané (1980, 1982); Marshall y Patterson (1981); Tonni et al. (1992, 1998) y Esteban y Nasif (1999). La barra cortada indica los registros aportados por este trabajo. Edad Mamífero: SC, Santacrucense; FR, "Friasense"; V, Miembro Vivero de la Formación Arroyo Chasicó (Chasiquense inferior); B, Miembro Las Barrancas de la Formación Arroyo Chasicó (Chasiquense superior); HU, Huayqueriense; MO, Montehermosense; CHA, Chapadmalalense; MA, Marplatense; EN, Ensenadense; LU, Lujanense.

Temporal distribution of the studied dasipodids taxa. The full bars indicate the records according to Scillato Yané (1980, 1982), Marshall and Patterson (1981), Tonni et al. (1992, 1998), and Esteban and Nasif (1999). The dashed bars indicate the contribution of this work. Land Mammal Age: SC, Santacrusian; FR, "Friasian"; V, Vivero Member of Arroyo Chasicó Formation (Lower Chasicoan); B, Las Barrancas Member of Arroyo Chasicó Formation (Upper Chasicoan); HU, Huayquerian; MO, Montehermosan; CHA, Chapadmalalan; MA, Marplatan; EN, Ensenadan; LU, Lujanian.

de los afloramientos fueron recolectados en superficie.

Todos los ejemplares analizados están depositados en la Cátedra de Geología Histórica de la Facultad de Ciencias Exactas y Naturales de la Universidad Nacional de La Pampa, Argentina, cuyo acrónimo es GHUNLPam.

\section{GEOLOGÍA}

La Formación Cerro Azul está constituida por tres asociaciones de facies, que de base a techo se interpretan: como una acumulación en un ambiente lacustre somero, sobre la que luego actuaron procesos edáficos; sobre esta facies se encuentra una asociación propia de un sistema eólico modificada por procesos edáficos y diagenéticos; finalmente, hay una tercera facies constituida por depósitos de un curso fluvial de escasa magnitud (Goin et al., en prensa).

La similitud litológica entre los depósitos de la Formación Cerro Azul y la Formación Arroyo Chasicó es muy marcada y, tal como lo expresan Linares et al. (1980), sólo es posible diferenciarlas cuando entre ambas se intercala la Formación Río Negro (departamento Caleu Caleu). Calmels et al. (1996) correlacionaron el Miembro Las Barrancas de la Formación Arroyo Chasicó
(Bondesio et al., 1980) con los afloramientos de la Formación Cerro Azul en el valle de Quehué. Montalvo et al. (1996) no avalan esta correlación teniendo en cuenta la fauna de mamíferos.

Los sedimentos loésicos que afloran en la provincia de La Pampa, particularmente los de la localidad Salinas Grandes de Hidalgo, fueron interpretados como parte de la "Formación Epecuén". El estudio sedimentológico detallado de las barrancas que bordean estas salinas avaló su inclusión en la Formación Cerro Azul (Goin et al., en prensa).

\section{SISTEMÁTICA}

Familia Dasypodidae Bonaparte, 1838

Subfamilia Euphractinae Pocock, 1924

Tribu Euphractini Pocock, 1924

Vetelia perforata Scillato Yané, 1977

Fig. 2 a - c

Material y procedencia geográfica

GHUNLPam 9184/4, 9184/19 y 9184/20 (Cerro de los Guanacos). Tres placas fijas completas, una de las cuales 
corresponde a la región anterior y las restantes a la lateroposterior del escudo pélvico.

\section{Observaciones}

Como es característico en este género, todas las figuras son globosas y la central subelíptica. $V$. perforata presenta numerosos forámenes pilíferos pequeños sobre el margen posterior, a diferencia de $V$. puncta Ameghino y V. gandhii Esteban y Nasif.

\section{Distribución}

$V$. perforata fue registrada en la Formación Collón Curá, "Friasense", Formación Huachipampa y Formación Arroyo Chasicó en las provincias de Chubut, Neuquén, San Juan, Buenos Aires y La Pampa, respectivamente (Scillato Yané, 1982). Recientemente se mencionó su presencia en los sedimentos que suprayacen a la Formación Arroyo Chasicó, en la provincia de Buenos Aires (Tonni et al., 1998) (Tabla 1).

\section{Macroeuphractus morenoi (Lydekker) Rovereto, 1914 \\ Fig. 2 d - g}

1920 Proeuphractus scalabrinii Ameghino, 677 (in part).

\section{Material y procedencia geográfica}

GHUNLPam 9164/1, 9164/2, 8317/1, 8317/94, 8317/98, $8317 / 99,8317 / 100,8317 / 101,8317 / 102,8317 / 103$ у $9165 / 2$ (Salinas Grandes de Hidalgo); GHUNLPam 9257/207 y 9257/226 (Loventué); GHUNLPam 2088 (Guatraché); GHUNLPam 9523/94, 9523/97, 8253/155, 8848/21, 9066/8, 8453/147 y 8453/177 (Telén); GHUNLPam 8198/95 (Quehué); GHUNLPam 5572/5, 2099/38, 2386/3 y 5568/1 (Laguna Chillhué). El material consiste en placas fijas y móviles completas y numerosos fragmentos de ambas.

\section{Observaciones}

Macroeuphractus morenoi posee las placas fijas con todas las figuras convexas y los surcos bien marcados. Las placas móviles poseen la figura central estrecha, muy elevada y abiselada, a diferencia de $M$. retusus Ameghino y $M$. outesi Ameghino.

\section{Distribución}

Esta especie está citada para la "Formación Epecuén" en las provincias de La Pampa y Buenos Aires y para el "Araucanense" en el valle de Santa María, provincia de Catamarca (Scillato Yané, 1982) (Tabla 1).

\section{Macrochorobates scalabrinii (Moreno y Mercerat) Scillato Yané, 1980 Fig. $2 \mathrm{~h}-\mathrm{i}$}

1914 Acantharodeia spicata Rovereto, 108-109.

\section{Material y procedencia geográfica}

GHUNLPam 2291/55, 2316/7 y 361/12 (Laguna Chillhué); GHUNLPam 8198/96 y 8198/100 (Quehué);
GHUNLPam 9523/95, 9058/34, 8453/135 y 8453/166 (Telén); GHUNLPam 8317/95 (Salinas Grandes de Hidalgo); GHUNLPam 9257/208 (Loventué). El material consiste en placas fijas completas y fragmentos de placas móviles, siendo las primeras más numerosas.

\section{Observaciones}

Las placas móviles de Macrochorobates scalabrinii tienen la figura central elevada y no se proyectan en punta hacia atrás de manera tan notoria como en $M$. chapalmalensis (Ameghino) Scillato Yané. A diferencia de lo que se observa en los ejemplares de $M$. chapalmalensis, las placas fijas poseen las figuras periféricas globosas.

\section{Distribución}

Macrochorobates scalabrinii se registra en la "Formación Epecuén", provincia de Buenos Aires y en el "Araucanense" del valle de Santa María, provincias de Tucumán y Catamarca (Marshall y Patterson, 1981, Scillato Yané, 1982). Recientemente ha sido citada para los sedimentos suprayacentes a la Formación Arroyo Chasicó, en la provincia de Buenos Aires (Tonni et al., 1998) (Tabla 1).

\section{Género Proeuphractus Ameghino, 1886}

\section{Proeuphractus sp.} Fig. 2 j - k

\section{Material y procedencia geográfica}

GHUNLPam 2099/3 y 8028/40 (Laguna Chillhué); GHUNLPam 8848/22 y 9058/48 (Telén); GHUNLPam 9688/48 (Quehué); GHUNLPam 2079 (Guatraché). El material consiste en fragmentos de placas móviles.

\section{Observaciones}

Las placas móviles presentan la figura central alargada y recta, delimitada por surcos anchos y de fondo cóncavo. Las perforaciones pilíferas son numerosas y están ubicadas en el margen posterior y en ambos márgenes laterales. Estos rasgos permiten asignar el material a Proeuphractus.

Este género cuenta con una sola especie, $P$. limpidus Ameghino. El nuevo material es de tamaño mayor (más del doble en largo y ancho). Esta diferencia permite inferir la presencia de una nueva especie para la Formación Cerro Azul, pero debido a que los restos son escasos y fragmentarios, se considera conveniente esperar a contar con ejemplares más completos para establecer un nuevo taxón.

\section{Distribución}

Proeuphractus está registrado en la Formación Arroyo Chasicó, en el "Hermosense típico" (Tonni et al., 1992), provincia de Buenos Aires y en el "Mesopotamiense" (Formación Ituzaingó), provincia de Entre Ríos (Scillato Yané, 1980, 1982) (Tabla 1). 

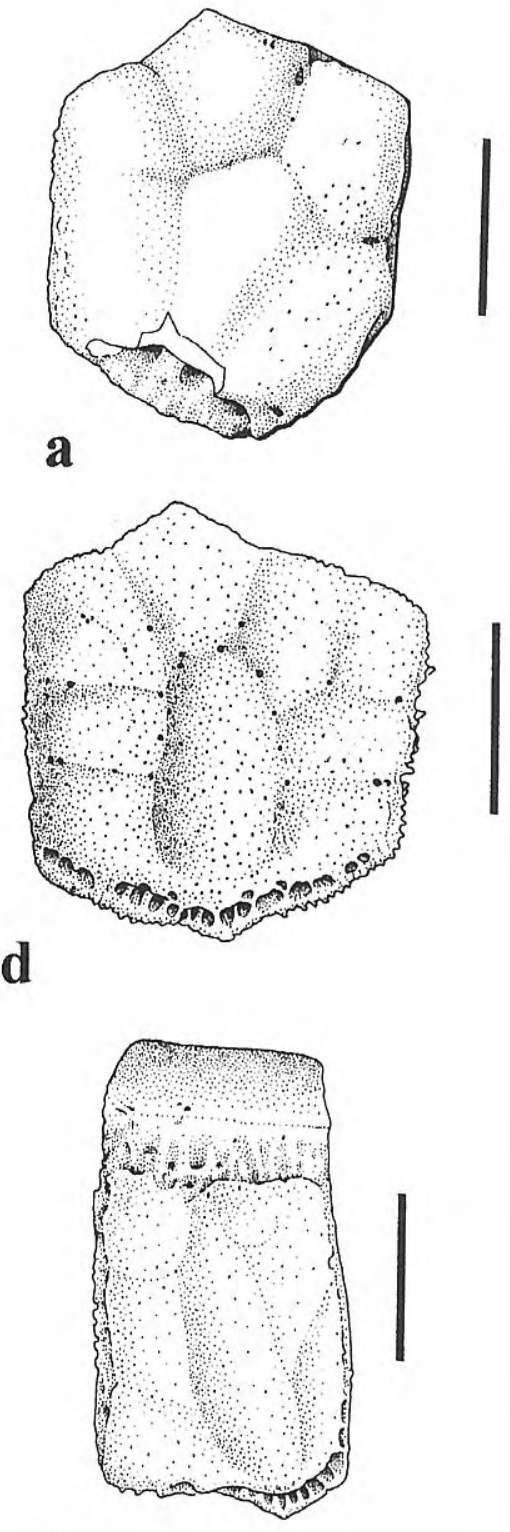

g

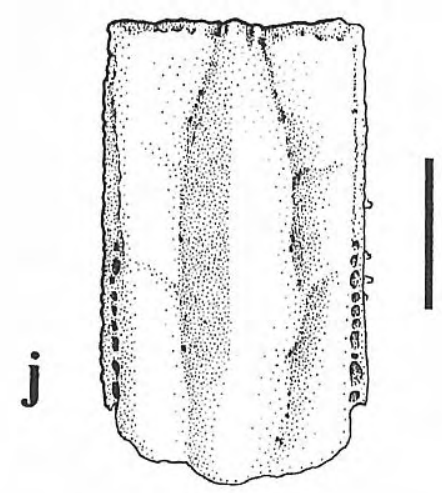

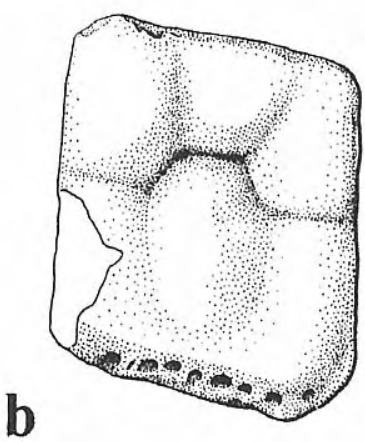

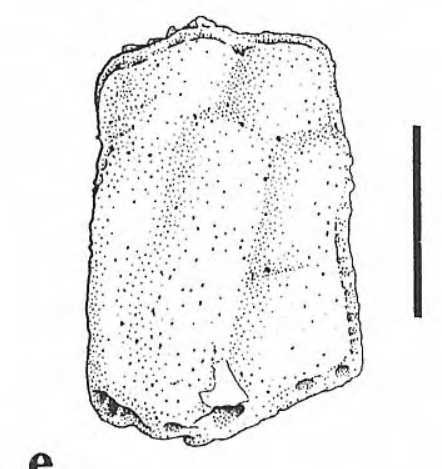

e

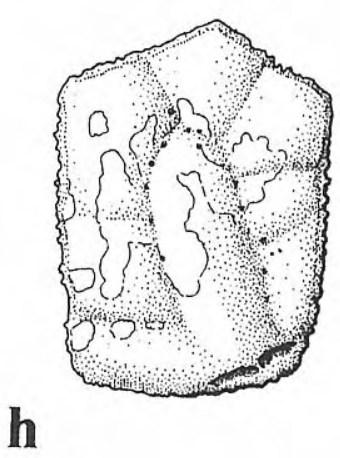

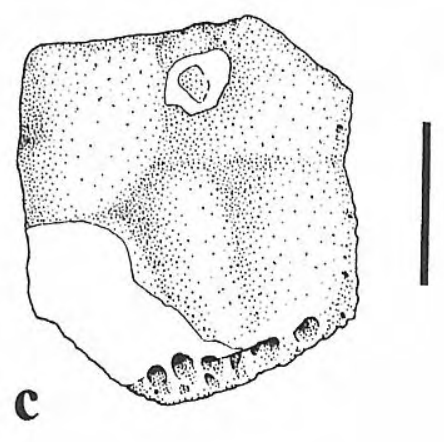
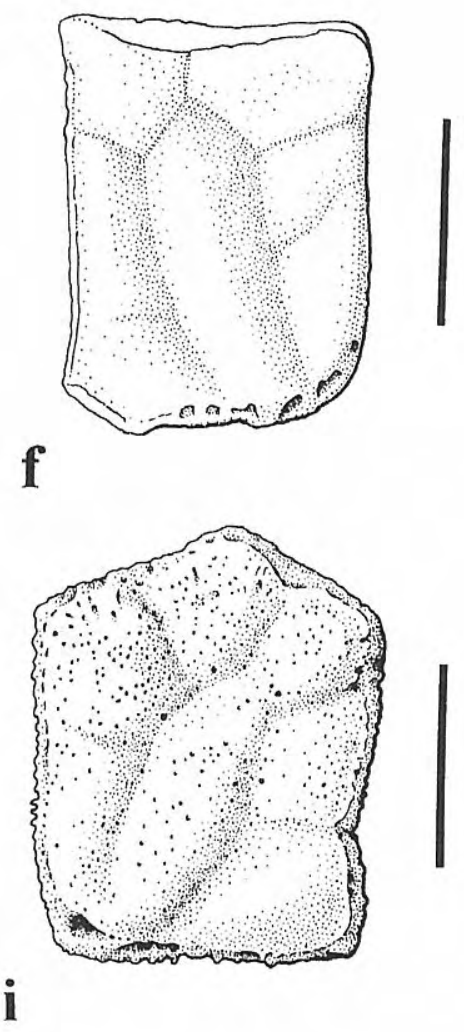

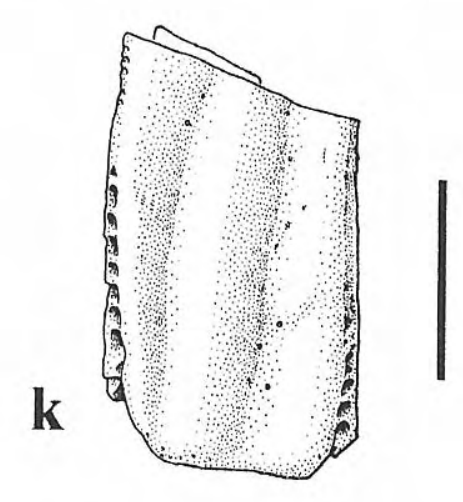

Figura 2. a-c Vetelia perforata, placas fijas: a (GHUNLPam 9184/19), b (GHUNLPam 9184/4) y c (GHUNLPam 9184/20). d-g Macroeuphractus morenoi, placas fijas: d (GHUNLPam 9523/94), e (GHUNLPam 9257/207), f (GHUNLPam 8848/21) y placa móvil: g (GHUNLPam 9257/226). h-i Macrochorobates scalabrinii, placas fijas: h (GHUNLPam 8453/135), i (GHUNLPam 8198/96). j-k Proeuphractus, fragmentos de placas móviles: j (GHUNLPam 2099/3), k (GHUNLPam 9688/48). Las escalas corresponden a $1 \mathrm{~cm}$.

$\boldsymbol{a}-\boldsymbol{c}$ Vetelia perforata, fixed scutes. $\boldsymbol{d}$-g Macroeuphractus morenoi, $\boldsymbol{d}$-f: fixed scutes, $g:$ movable scute. $\boldsymbol{h}-\boldsymbol{i}$ Macrochorobates scalabrinii, fixed scutes. $\boldsymbol{j}$ - $k$ Proeuphractus $s p$., movable scutes fragments. Scale bars equal $1 \mathrm{~cm}$. 


\section{Zaedyus pichiy (Desmarest) Osgood, 1919 Fig. 3 a}

\section{Material y procedencia geográfica \\ GHUNLPam 8419/3 y 8320/9 (Telén); GHUNLPam 8606/45, 5433/9 y 5433/13 (Laguna Chillhué); GHUNLPam 9688/49 y 9688/52 (Quehué); GHUNLPam 5550/1 (Cerro de la Bota). El material consiste en placas fijas y móviles completas y fragmentos de ambas.}

\section{Observaciones}

Las placas móviles presentan la figura central de lados paralelos, estrecha y convexa. Las figuras laterales son también convexas y están divididas en dos o tres figuritas menores, a veces con los surcos que las dividen muy superficiales, particularmente los anteriores. Las placas fijas tienen la figura central y las periféricas convexas. Los forámenes pilíferos, en ambos tipos de placas, son muy pequeños y están ubicados en el margen posterior. De modo coincidente con lo descrito por Vizcaíno y Bargo (1993), el foramen más grande coincide con la figura central. Si bien todas las placas tienen características morfológicas similares, hay un grupo que se diferencia por su tamaño menor, similar al de Zaedyus minimus (Ameghino) (placas fijas, largo: 8,5-9 mm, ancho: 5-6 $\mathrm{mm}$; placas móviles, largo: $12,5 \mathrm{~mm}$, ancho: 4,5-5 mm).

\section{Distribución}

Z. pichiy se ha registrado en las Formaciones ?Miramar, Ensenada, Buenos Aires y Luján en la provincia de Buenos Aires (Scillato Yané, 1982); actualmente se encuentra distribuida desde el sur de Mendoza, La Pampa y Buenos Aires hasta el extremo austral del país (Tabla 1).

\section{Gen. nov. "A" Scillato Yané, 1982 Fig. 3 b}

\section{Material y procedencia geográfica}

GHUNLPam 9892, 9893 y 9894 (Salinas Grandes de Hidalgo); GHUNLPam 2316/17 (Laguna Chillhué). El material consiste en tres placas fijas y una placa móvil.

\section{Observaciones}

La placa móvil presenta las figuras periféricas claramente divididas en figuras menores. En las placas móviles y fijas, las figuras periféricas son más convexas y con los forámenes pilíferos más grandes que en Zaedyus.

Las características del material concuerdan con las de un género y especie nuevos descritos por Scillato Yané en su Tesis Doctoral (1982, págs. 109-110).

\section{Distribución}

Este taxón tiene registro en la Formación Monte Hermoso, provincia de Buenos Aires (Scillato Yané, 1982) (Tabla 1).

\section{Chorobates villosissimus (Rovereto) Reig, 1958} Fig. $3 \mathrm{c}-\mathrm{d}$

1914 Proeuphractus minor Rovereto, 224.

\section{Material y procedencia geográfica}

GHUNLPam 8317/3, 8317/204, 8317/205, 8317/206, $8317 / 207,8317 / 208,8317 / 209,8317 / 210,8317 / 211$, $8317 / 212$ y $8317 / 213$ (Salinas Grandes de Hidalgo); GHUNLPam 5550/2, 5550/4 y 5550/5 (Cerro de la Bota); GHUNLPam 9523/96, 8453/163, 8253/57, 8848/23 y 8848/24 (Telén); GHUNLPam 9257/204, 9257/209, 9257/210, 9257/211, 9257/212 y 9257/213 (Loventué); GHUNLPam 2389/1, 361/40, 8606/30 y 5433/10 (Laguna Chillhué); GHUNLPam 8198/97, 8198/101, 8198/102 y 8198/103 (Quehué). El material consiste en numerosas placas fijas y móviles completas y fragmentos de ambas.

\section{Observaciones}

Las placas móviles y fijas presentan la figura central poco elevada y poco desviada hacia el lado externo, como es característico de Chorobates villosissimus. Chorobates recens (Ameghino) Reig, en cambio, presenta estos rasgos muy marcados.

\section{Distribución}

C. villosissimus está registrada en el "Hermosense típico" (Tonni et al., 1992), el "Araucanense", la "Formación Epecuén" y la Formación Tunuyán (Huayquerías de San Carlos), en las provincias de Buenos Aires, Catamarca, La Pampa y Mendoza, respectivamente (Scillato Yané, 1982) (Tabla 1).

\section{Tribu Eutatini Bordas, 1933}

\section{Chasicotatus ameghinoi Scillato-Yané, 1977} Fig. 3 e - f

\section{Material y procedencia geográfica}

GHUNLPam 8198/98, 8198/106, 8198/107, 8198/108, 8198/109, 8198/110,8198/111,8198/112,8198/113, 8198/114 y 9688/51 (Quehué); GHUNLPam 2373/1 (Bajo Giuliani); GHUNLPam 2111/2, 2111/3 y 2111/4 (Naicó); GHUNLPam 9064/15, 9059/11, 9059/40, 8252/3, 8253/105, $8452 / 2,8452 / 3,8452 / 5,8452 / 6,8452 / 11,8452 / 15,8452 / 19$, $8452 / 20,8452 / 21,8452 / 24,8452 / 25,8452 / 76,9167 / 7$, $8848 / 4,8848 / 5,8848 / 25$ y $8848 / 26$ (Telén); GHUNLPam $8317 / 96,8317 / 122,8317 / 123,8317 / 124$ y 8317/125 (Salinas Grandes de Hidalgo); GHUNLPam 5433/1, 5433/15, $8034 / 15,8606 / 15,2386 / 17,2386 / 23$ y 2163/29 (Laguna Chillhué); GHUNLPam 9257/2, 9257/205, 9257/227, 9257/228, 9257/229, 9257/230, 9257/231, 9257/232, 9257/233, 9257/234, 9257/235, 9257/236, 9257/237, 9257/238, 9257/239, 9257/240, 9257/241, 9257/242, 9257/243, 9257/244 y 9257/245 (Loventué); GHUNLPam 9935 (Cerro de los Guanacos). El material consiste en numerosas placas fijas, seis placas móviles completas y fragmentos de ambas.

\section{Observaciones}

Las placas móviles presentan una figura central alargada con el extremo posterior aguzado. Las placas fijas tienen todas las figuras globosas y la central forma 

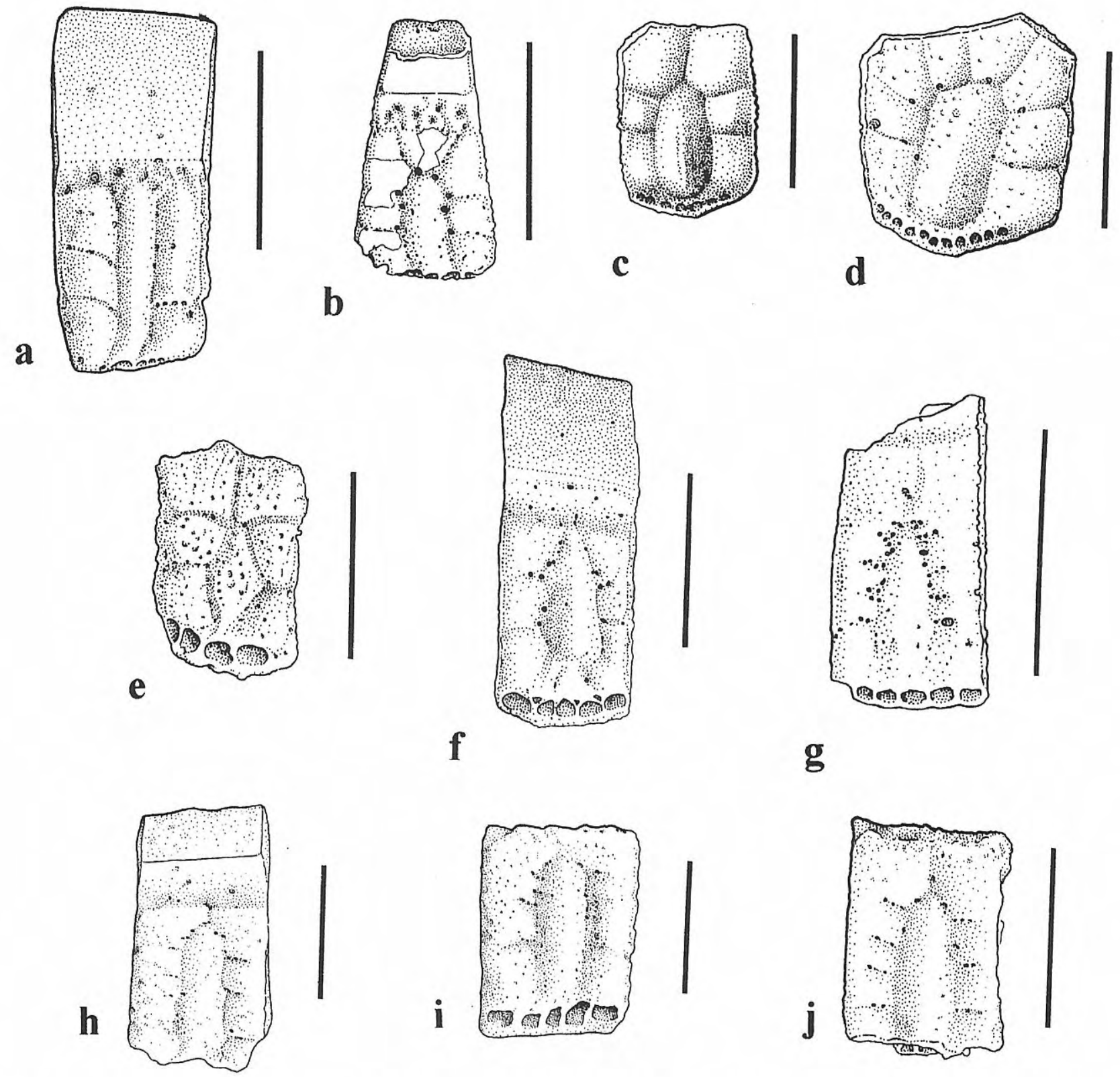

Figura 3. a Zaedyus pichiy, placa móvil (GHUNLPam 12528). b Gen. nov. "A” Scillato Yané, 1982), placa móvil (GHUNLPam 2316/17). c-d Chorobates villosissimus, placas fijas: c (GHUNLPam 8253/57) y d (GHUNLPam 8453/163). e-f Chasicotatus ameghinoi, placa fija: e (GHUNLPam 8198/98) y placa móvil: f (GHUNLPam 8198/106). g Doellotatus inornatus, fragmento de placa móvil (GHUNLPam 5433/6). h-j Doellotatus chapadmalensis, fragmentos de placas móviles: h (GHUNLPam 8317/105), i (GHUNLPam 8317/5) y j (GHUNLPam 8317/97). Las escalas corresponden a $1 \mathrm{~cm}$.

a Zaedyus pichiy, movable scute. b Gen. nov. "A" Scillato Yané (1982), movable scute. c-d Chorobates villosissimus, fixed scutes. $\boldsymbol{e}$-f Chasicotatus ameghinoi, fixed scute: $e$, and movable scute: $f$. $g$ Doellotatus inornatus, movable scute fragment. $\boldsymbol{h}$-j Doellotatus chapadmalensis, movable scutes fragments. Scale bars equal $1 \mathrm{~cm}$.

una carena elevada. Los forámenes pilíferos son de gran tamaño en número variable de tres a cinco.

\section{Distribución}

Chasicotatus ameghinoi fue citada para la Formación Arroyo Chasicó en la provincia de Buenos Aires (Scillato Yané, 1982) y para la Formación Chiquimil (Miembro A= Miembro El Jarillal) en la provincia de Catamarca (Esteban y Nasif, 1999) (Tabla 1).
Doellotatus inornatus (Rovereto) Bordas, 1932 Fig. $3 \mathrm{~g}$

\section{Material y procedencia geográfica}

GHUNLPam 8452/77, 8452/78, 8452/79, 9065/23 y 9067/109 (Telén); GHUNLPam 8198/99, 8198/104, 8198/105, 9688/50, 9688/52, 9688/53 y 9688/54 (Quehué); GHUNLPam 5550/3 (Cerro de la Bota); GHUNLPam $8028 / 43,5433 / 6,5433 / 12,5433 / 16,2389 / 2,5609 / 6,5609 / 8$ 
y 8404/3 (Laguna Chillhué); GHUNLPam 9257/5, 9257/206, 9257/209, 9257/210, 9257/211, 9257/212, 9257/213, 9257/214, 9257/215, 9257/216, 9257/217, 9257/218, 9257/219, 9257/220, 9257/221, 9257/222, 9257/223, 9257/224 y 9257/225 (Loventué). El material consiste en numerosas placas móviles y fijas completas y fragmentos de ambas.

\section{Observaciones}

Doellotatus inornatus presenta las placas fijas y móviles con la figura central elevada, si bien en algunas placas móviles la figura central es plana en su parte anterior.

\section{Distribución}

Esta especie se registró en la Formación Monte Hermoso, "Hermosense típico" (Tonni et al., 1992) y en la Formación Irene en la provincia de Buenos Aires (Scillato Yané, 1982) (Tabla 1).

\section{Doellotatus chapadmalensis Bordas, 1933 Fig. $3 \mathrm{~h}-\mathrm{j}$}

\section{Material y procedencia geográfica}

GHUNLPam 8317/2, 8317/5, 8317/97, 8317/114, $8317 / 115,8317 / 116,8317 / 117,8317 / 118,8317 / 119$, $8317 / 120$ y $8317 / 121$ (Salinas Grandes de Hidalgo). El material consiste en fragmentos de placas móviles y una placa fija casi completa.

\section{Observaciones}

Doellotatus chapadmalensis presenta placas de tamaño mayor que $D$. inornatus, con la figura central más ancha y plana que la de esa especie.

\section{Distribución}

Doellotatus chapadmalensis se registró en el "Hermosense típico" (Tonni et al., 1992), en la Formación Irene y en la Formación Chapadmalal, provincias de Buenos Aires y La Pampa (Scillato Yané, 1982) (Tabla 1).

\section{DISCUSIÓN Y CONCLUSIONES}

Los dasipódidos estudiados en este trabajo proceden, en su mayoría, de los niveles lacustres y eólicos de la Formación Cerro Azul. En la localidad Cerro de la Bota, en cambio, las sedimentitas portadoras corresponden a una facies interpretada como de origen fluvial (Goin et al., en prensa).

Chasicotatus ameghinoi, Macrochorobates scalabrinii, Macroeuphractus morenoi y Chorobates villosissimus son las especies que se encuentran más frecuentemente asociadas, registrándose en cinco de las localidades muestreadas: Laguna Chillhué, Telén, Loventué, Quehué y Salinas Grandes de Hidalgo. En las cuatro primeras localidades se encuentra, además, Doellotatus inornatus, en tanto que en Laguna Chillhué, Telén y Quehué se suma a estos taxones la presencia de Proeuphractus (Tabla 2).
Vetelia perforata, Chasicotatus ameghinoi y Proeuphractus fueron mencionadas por Bondesio et al. (1980) para la Formación Arroyo Chasicó (estratotipo del Chasiquense). De estos taxones, V. perforata está presente en el Miembro Vivero y el Miembro Las Barrancas, los restantes taxones sólo se registran en el miembro superior (Miembro Las Barrancas). Tonni et al. (1998) establecen la Biozona de Chasicotatus ameghinoi en el Miembro Las Barrancas como base bioestratigráfica del Chasiquense superior (Barranquense). Esta especie ha sido citada, también, para el miembro superior (Miembro $\mathrm{A}=$ Miembro El Jarillal) (Muruaga, 1998) de la Formación Chiquimil en el valle de Hualfín, provincia de Catamarca (Esteban y Nasif, 1999). Proeuphractus fue citado en el "Mesopotamiense" (Formación Ituzaingó) (Scillato Yané, 1982); sin embargo, el material sobre el que se fundamenta el género y el material referido fueron recolectados a fines del siglo XIX - principios del XX y no cuentan con procedencia geográfica y estratigráfica precisa. Se postuló que el "Mesopotamiense" podría involucrar más de una de las Edades Mamífero reconocidas para la Pampasia (Chasiquense, Huayqueriense y Montehermosense) o que los restos podrían representar una mezcla de ejemplares procedentes de niveles no coetáneos (Scillato Yané, 1975).

Macrochorobates scalabrinii, Macroeuphractus morenoi y Chorobates villosissimus fueron citadas para la "Formación Epecuén", en las provincias de Buenos Aires y La Pampa y para el "Araucanense" del valle de Santa María, en la provincia de Catamarca (Scillato Yané, 1982). El material del "Araucanense" carece de datos geográficos y estratigráficos precisos, por lo que podría proceder de una u otra de las formaciones neógenas que afloran extensamente en el valle (formaciones Las Arcas, Chiquimil, Andalhuala o Corral Quemado). Sólo Chorobates scalabrinii (= Macrochorobates scalabrinii) es mencionada por Marshall y Patterson (1981) como procedente de las unidades XVII, XX, y 24 de los perfiles realizados en las localidades de Entre Ríos (=Chiquimil) y Puerta de Corral Quemado, respectivamente. Estos niveles son referidos a la Formación Andalhuala (Bossi et al., 1987) de edad Huayqueriense. M. scalabrinii fue recolectado, también, en los sedimentos suprayacentes a la Formación Arroyo Chasicó, en la provincia de Buenos Aires, motivando el establecimiento de la Biozona de Macrochorobates scalabrinii asignada al Huayqueriense temprano (Tonni et al., 1998).

Doellotatus inornatus y Gen. nov. "A" Scillato Yané, 1982 se registraron en la Formación Monte Hermoso y la primera, además, en la Formación Irene de la provincia de Buenos Aires, sin datos más precisos sobre su procedencia. Doellotatus chapadmalensis fue citada para las formaciones Irene y Chapadmalal. Recientemente fue mencionada para la "Formación" Vorohué en la costa atlántica bonaerense (Scillato Yané et al., 1995). Tonni et al. (1992) consideran conveniente no referir la "Formación" Irene al Montehermosense hasta que existan colecciones paleontológicas con buen control estratigráfico. 


\begin{tabular}{|c|c|c|c|c|c|c|c|c|c|c|}
\hline Taxones $\quad$ Localidad & $\begin{array}{l}\text { Laguna } \\
\text { Chillhué }\end{array}$ & Telén & Quehué & Loventué & $\begin{array}{l}\text { Cerro de } \\
\text { la Bota }\end{array}$ & Naicó & Guatraché & $\begin{array}{c}\text { Bajo } \\
\text { Giuliani }\end{array}$ & $\begin{array}{c}\text { Cerro de } \\
\text { los Guanacos }\end{array}$ & $\begin{array}{l}\text { Salinas Grandes } \\
\text { de Hidalgo }\end{array}$ \\
\hline Vetelia perforata & & & & & & & & & $\mathrm{x}$ & \\
\hline Macroeuphractus morenoi & $\mathrm{x}$ & $\mathrm{x}$ & $\mathrm{x}$ & $x$ & & & $\mathrm{x}$ & & & $\mathrm{x}$ \\
\hline Macrochorobates scalabrinii & $\mathrm{x}$ & $\mathrm{x}$ & $\mathrm{x}$ & $\mathrm{x}$ & & & & & & $\mathrm{x}$ \\
\hline Proeuphractus sp. & $x$ & $x$ & $x$ & & & & $\mathrm{x}$ & & & \\
\hline Zaedyus pichiy & $\mathrm{x}$ & $\mathrm{x}$ & $\mathrm{x}$ & & $\mathrm{x}$ & & & & & \\
\hline Género nov."A" Scillato Yané & & & $\mathrm{x}$ & & & & & & & $\mathrm{x}$ \\
\hline Chorobates villosissimus & $\mathrm{x}$ & $x$ & $\mathrm{x}$ & $\mathrm{x}$ & $x$ & & & & & $\mathrm{x}$ \\
\hline Chasicotatus ameghinoi & $\mathrm{x}$ & $\mathrm{x}$ & $\mathrm{x}$ & $\mathrm{x}$ & & $\mathrm{x}$ & & $\mathrm{x}$ & $\mathrm{x}$ & $\mathrm{x}$ \\
\hline Doellotatus inornatus & $\mathrm{x}$ & $\mathrm{x}$ & $\mathrm{x}$ & $\mathrm{x}$ & $\mathrm{x}$ & & & & & \\
\hline Doellotatus chapadmalensis & & & & & & & & & & $x$ \\
\hline
\end{tabular}

Tabla 2. Distribución de los taxones de dasipódidos en las localidades de la provincia de La Pampa, Argentina. Distribution of the dasipodids taxa in the localities from La Pampa province, Argentina.

Como se desprende de lo detallado en los párrafos anteriores, de los dasipódidos dados a conocer en este trabajo y que en otras regiones del país han sido reseñados como exclusivos del Huayqueriense, sólo Macrochorobates scalabrinii cuenta con procedencia estratigráfica precisa. Los restantes se han registrado en edades previas o posteriores al Huayqueriense. Sin embargo, teniendo en cuenta otros grupos de mamíferos, diversos autores han referido numerosos afloramientos de la Formación Cerro Azul al Huayqueriense (Mioceno tardío) (Verzi et al., 1994, 1995, 1999; Montalvo et al., 1996, 1998), si bien Verzi (1999) considera los niveles aflorantes en Cerro de la Bota como asignables a la edad Chasiquense, basándose en los restos de roedores octodóntidos.

En este marco, el nuevo registro de dasipódidos permite contrastar algunas inferencias previamente planteadas. Scillato Yané (1977 y 1982) señaló la escasez de Pampatheriinae para el Huayqueriense en la Pampasia. Aunque el nuevo material de dasipódidos de la Formación Cerro Azul es abundante y diverso (10 géneros y 8 especies) no se reconocen aún pampaterinos.

Se ha mencionado la abundancia de Eutatini en el Huayqueriense de la Pampasia (Scillato Yané, 1975 y 1977). El registro de la Formación Cerro Azul permite convalidar esta idea ya que Chasicotatus ameghinoi está presente en el $50 \%$ de las localidades estudiadas, Doellotatus inornatus en el $40 \%$ y D. chapadmalensis en el $10 \%$ de las mismas.

Scillato Yané (1977) manifiesta la ausencia de Proeuphractus durante el Huayqueriense en la Pampasia. Entre los materiales estudiados para este trabajo, se reconoce Proeuphractus en la Formación Cerro Azul.

Chasicotatus ameghinoi, Macrochorobates scalabrinii, Zaedyus pichiy, Vetelia perforata,
Doellotatus inornatus, Doellotatus chapadmalensis, Gen. nov. "A" Scillato Yané (1982) y Proeuphractus se reconocen por vez primera en la Formación Cerro Azul (Huayqueriense, Mioceno tardío) de la provincia de La Pampa.

El registro de Chasicotatus ameghinoi en la Formación Cerro Azul es el más moderno para este taxón. El de Zaedyus pichiy, Gen. nov. A Scillato Yané (1982), Doellotatus inornatus y Doellotatus chapadmalensis extiende la antigüedad de estos dasipódidos hasta el Huayqueriense.

Se infiere la presencia de una nueva especie de Proeuphractus en la Formación Cerro Azul por el tamaño mucho mayor (más del doble en longitud y anchura) del material estudiado con respecto a la especie $P$. limpidus.

\section{AGRADECIMIENTOS}

Agradecemos al Licenciado Enrique Guanuco, quien realizó los dibujos que se exponen en este trabajo y al Licenciado Daniel Ruíz Holgado por su colaboración en la elaboración de los cuadros.

\section{BIBLIOGRAFÍA}

Ameghino, F. 1886. Contribuciones al conocimiento de los mamíferos fósiles de los terrenos terciarios antiguos del Paraná. Boletín de la Academia Nacional de Ciencias, Córdoba, 9 (1-2), 5-228.

Ameghino, F. 1920. Sur les édentés fossiles de l'Argentine. (Examen critique, révision et correction de l'ouvrage de Mr. R. Lydekker: "The extinct edentates from Argentina", etc.). Obras completas y correspondencia 
científica de Florentino Ameghino, La Plata, 11, 447909.

Bondesio, P., Laza, J., Scillato Yané, G. J., Tonni, E. y Vucetich, M. G. 1980. Estado actual del conocimiento de los vertebrados de la Formación Arroyo Chasicó (Plioceno temprano) de la provincia de Buenos Aires. II Congreso Argentino de Paleontología y Bioestratigrafía y I Congreso Latinoamericano de Paleontología, Buenos Aires, 3, 101-127.

Bordas, A. F. 1932. Proposición de un nuevo género para Eutatus inornatus. Physis, 11 (38), 167-168.

Bordas, A. F. 1933. Notas sobre los Eutatinae. Nueva subfamilia extinguida de Dasypodidae. Anales del Museo Nacional de Historia Natural, 37, 583-614.

Bossi, G. E., Ovejero, R. y Strecker, M. 1987. Correlación entre los perfiles del Terciario Superior en la Puerta de Corral Quemado - Hualfín y de Entre Ríos (Chiquimil) provincia de Catamarca, Argentina. Actas del Décimo Congreso Geológico Argentino, Tucumán, 2, 117-120.

Calmels, A. P., Visconti, G., Carballo, O. y Sbrocco, J. 1996. Los sedimentos del Pleistoceno tardío-Holoceno encauzados en el valle de Quehué, provincia de La Pampa. Actas VI Reunión Argentina de Sedimentología. I Simposio de Arcillas, Bahía Blanca, 135-140.

Esteban, G. y Nasif, N. 1999. Mamíferos fósiles de la Formación Chiquimil (Mioceno tardío), provincia de Catamarca, Argentina. Bioestratigrafía. Ameghiniana, 36, Suplemento, 11R.

Esteban, G., Nasif, N. y Montalvo, C. 1996. Nuevos registros de Dasypodidae (Edentata) del Terciario tardío (Huayqueriense) de la provincia de La Pampa. Ameghiniana, 33, 464.

Goin, F. J. y Montalvo, C. I. 1988. Revisión sistemática y reconocimiento de una nueva especie del género Thylatheridium Reig (Marsupialia, Didelphidae). Ameghiniana, 25, 161-167.

Goin, F. J., Montalvo, C. I. y Visconti, G. 1997. Los marsupiales de la Formación Cerro Azul (Mioceno tardío), provincia de La Pampa, Argentina. Ameghiniana, 34, 536.

Goin, F. J., Montalvo, C. I. y Visconti, G. (en prensa). Los marsupiales (Mammalia) del Mioceno superior de la Formación Cerro Azul (provincia de La Pampa, Argentina). Estudios Geológicos.

Linares, E., Llambías, E. y Latorre, C. 1980. Geología de la provincia de La Pampa, República Argentina y Geocronología de sus rocas metamórficas y eruptivas. Revista de la Asociación Geológica Argentina, 35, 87146.

Marshall, L. G. and Patterson, B. 1981. Geology and geochronology of the mammal-bearing Tertiary of the Valle de Santa María and Río Corral Quemado, Catamarca Province, Argentina. Fildeana Geology (1321), New Series, 9, 1-78.

Montalvo, C. I. y Casadío, S. 1988. Presencia del género Palaeoctodon (Rodentia, Octodontidae) en el Huayqueriense (Mioceno tardío) en la Provincia de La Pampa. Ameghiniana, 25, 111-114.

Montalvo, C. I., Visconti, G., Púgener, L. A. y Cardonatto, M. C. 1995. Mamíferos de Edad Huayqueriense
(Mioceno tardío), Laguna Chillhué, provincia de La Pampa. Actas IV Jornadas Geológicas y Geofísicas Bonaerenses, Junín, Buenos Aires, 1, 73-79.

Montalvo, C. I., Cardonatto, M. C., Visconti, G., Verzi, D. H. y Vucetich, M. G. 1996. Vertebrados de la Formación Cerro Azul (Mioceno tardío) del Valle de Quehué, provincia de La Pampa, Argentina. Actas VI Jornadas Pampeanas de Ciencias Naturales, Santa Rosa, 159-165.

Montalvo, C. I., Verzi, D. H., Vucetich, M. G. y Visconti, G. 1998. Nuevos Eumysopinae (Rodentia, Echimyidae) de la Formación Cerro Azul (Mioceno tardío) de La Pampa, Argentina. Actas V Jornadas Geológicas y Geofísicas Bonaerenses, Mar del Plata, 1, 57-64.

Muruaga, C. M. 1998. Estratigrafía y sedimentología del Terciario superior de la Sierra de Hualfín, entre las localidades de Villavil y San Fernando, provincia de Catamarca. Tesis Doctoral, Facultad de Ciencias Naturales e Instituto Miguel Lillo. Universidad Nacional de Tucumán (Inédita), 1-162.

Ortega Hinojosa, E. 1967. Descripción de los restos de ún Scelidotheriinae (Edentata, Mylodontidae) de edad Huayqueriense. Algunas consideraciones en torno a la filogenia de los Scelidotheriinae. Ameghiniana, 5, 109120.

Osgood, W. H. 1919. Names of some South American mammals. Journal of Mammalogy, 1, 33-36.

Pascual, R. y Bocchino, A. 1963. Un nuevo Borhyaeninae (Marsupialia) del Plioceno medio de Hidalgo (La Pampa). Ameghiniana, 3, 97-107.

Pascual, R. y Bondesio, P. 1982. Un roedor Cardiatheriinae (Hydrochoeridae) de la Edad Huayqueriense (Mioceno tardío) de La Pampa. Sumario de los ambientes terrestres en la Argentina durante el Mioceno. Ameghiniana, 19, 19-35.

Pascual, R., Pisano, J. y Ortega, E. 1965. Un nuevo Octodontidae (Rodentia, Caviomorpha) de la Formación Epecuén (Plioceno medio) de Hidalgo (Provincia de La Pampa). Ameghiniana, 4, 19-30.

Reig, O. A. 1958. Notas para una actualización del conocimiento de la fauna de la Formación Chapadmalal. I. Lista faunística preliminar. Acta Geológica Lilloana, 2, 241-253.

Rovereto, C. 1914. Los estratos araucanos y sus fósiles. Anales del Museo Nacional de Historia Natural de Buenos Aires, 25, 1-250.

Scillato Yané, G. J. 1975. Nuevo género de Dasypodidae (Edentata-Xenarthra) del Plioceno de Catamarca (Argentina). Algunas consideraciones filogenéticas y zoogeográficas sobre los Euphractini. Actas I Congreso Argentino de Paleontología y Bioestratigrafía, San Miguel de Tucumán, 2, 449-461.

Scillato Yané, G. J. 1977. Notas sobre los Dasypodidae (Mammalia, Edentata) del Plioceno del territorio argentino. I. Los restos de Edad Chasiquense (Plioceno inferior) del sur de la provincia de Buenos Aires. Ameghiniana, 14, 133-144.

Scillato Yané, G. J. 1980. Catálogo de los Dasypodidae fósiles (Mammalia, Edentata) de la República Argentina. Actas II Congreso Argentino de Paleontología y Bioestratigrafía y I Congreso Latinoamericano de 
Paleontología, Buenos Aires, 3, 7-36.

Scillato Yané, G. J. 1982. Los Dasypodidae (MammaliaEdentata) del Plioceno y Pleistoceno de Argentina. Tesis Doctoral. Facultad de Ciencias Naturales y Museo, Universidad Nacional de La Plata (Inédita), 1-159.

Scillato Yané, G. J., Carlini, A. A., Vizcaíno, S. F. y Ortiz Jaureguizar, E. 1995. Los Xenartros. In: Evolución biológica y climática de la región pampeana durante los últimos cinco millones de años. Un ensayo de correlación con el mediterraneo occidental (Eds. M. T. Alberdi, G. Leone y E.P. Tonni). Museo Nacional de Ciencias Naturales, Madrid, Monografías, 12, 183-209.

Tonni, E. P., Prado, J. L., Fidalgo, F. y Laza, J. L. 1992. El $\mathrm{Piso/Edad} \mathrm{Montehermosense} \mathrm{(Plioceno)} \mathrm{y} \mathrm{sus}$ mamíferos. Actas III Jornadas Geológicas Bonaerenses, La Plata, 113-118.

Tonni, E. P., Scillato Yané, G. J., Cione, A. L. y Carlini, A. 1998. Bioestratigrafía del Mioceno continental en el curso inferior del arroyo Chasicó, provincia de Buenos Aires. Actas VII Congreso Argentino de Paleontología y Bioestratigrafía, Bahía Blanca, 135.

Verzi, D. H. 1999. Origen and differentation of the Ctenomyinae rodents (Caviomorpha, Octodontidae). Acta Theriologica, 44, 263-282.

Verzi, D. H., Montalvo, C. I. y Vucetich, M. G. 1990. La sistemática de los más antiguos Ctenomyinae (Rodentia, Octodontidae) del Mioceno Superior. Ameghiniana, 27, 391.

Verzi, D. H., Montalvo, C. I. y Vucetich, M. G. 1991. Nuevos restos de Xenodontomys simpsoni Kraglievich y la sistemática de los más antiguos Ctenomyinae (Rodentia, Octodontidae). Ameghiniana, 28, 325-331.

Verzi, D. H., Vucetich, M. G. y Montalvo, C. I. 1993. Dos nuevos Eumysopinae (Rodentia, Echimyidae) de la Formación Cerro Azul (Mioceno tardío) de La Pampa y consideraciones acerca de la historia de la subfamilia. Actas V Jornadas Pampeanas de Ciencias Naturales, Santa Rosa, 50.

Verzi, D. H., Vucetich, M. G. and Montalvo, C. I. 1994. Octodontid-like Echimyidae (Rodentia): an Upper Miocene episode in the radiation of the family. Paleovertebrata, 23, 199-210.

Verzi, D. H., Vucetich, M. G. y Montalvo, C. I. 1995. Un nuevo Eumysopinae (Rodentia, Echimyidae) del Mioceno tardío de la provincia de La Pampa y consideraciones sobre la historia de la subfamilia. Ameghiniana, 32, 191-195.

Verzi, D. H., Montalvo, C. I. y Vucetich, M. G. 1996. Estado sistemático, afinidades y significado evolutivo del octodóntido Neophanomys biplicatus del Mioceno tardío-Plioceno temprano? de Argentina (Rodentia, Caviomorpha). Ameghiniana, 33, 472.

Verzi, D. H., Montalvo, C. I. y Vucetich, M. G. 1999. Afinidades y significado evolutivo de Neophanomys biplicatus (Rodentia, Octodontidae) del Mioceno tardíoPlioceno temprano de Argentina. Ameghiniana, 36, 8390.

Vizcaíno, S. F. y Bargo, M. S. 1993. Los armadillos (Mammalia, Dasypodidae) de La Toma (partido de Coronel Pringles) y otros sitios arqueológicos de la provincia de Buenos Aires. Consideraciones paleoambientales. Ameghiniana, 30, 435-443.

Visconti, G. y Montalvo, C. I. 1990. Mamíferos del Mioceno tardío en sedimentos redepositados de la Formación Cerro Azul. Actas IV Jornadas Pampeanas de Ciencias Naturales, Santa Rosa, 14.

Zetti, J. 1972. Los mamíferos fósiles de Edad Huayqueriense (Plioceno medio) de la región pampeana. Tesis Doctoral. Facultad de Ciencias Naturales y Museo, Universidad Nacional de La Plata. (Inédita), 1-122.

Manuscrito recibido: 11 de mayo, 2000 Manuscrito aceptado: 14 de septiembre, 2000 